\title{
Frontières
}

\section{Bibliographie indicative sur la pensée de sa mort}

\section{Johanna Toffanello et Patrick Bergeron}

Volume 19, numéro 2, printemps 2007

Penser sa mort?

URI : https://id.erudit.org/iderudit/017512ar

DOI : https://doi.org/10.7202/017512ar

Aller au sommaire du numéro

Éditeur(s)

Université du Québec à Montréal

ISSN

1180-3479 (imprimé)

1916-0976 (numérique)

Découvrir la revue

Citer ce document

Toffanello, J. \& Bergeron, P. (2007). Bibliographie indicative sur la pensée de sa mort. Frontières, 19(2), 82-83. https://doi.org/10.7202/017512ar d'utilisation que vous pouvez consulter en ligne.

https://apropos.erudit.org/fr/usagers/politique-dutilisation/ 


\section{BIBLIOGRAPHIE INDICATIVE SUR LA PENSÉE DE SA MORT}

Johanna Toffanello et Patrick Bergeron

AGGOUN, Atmane (2006). Les musulmans face à la mort en France, Paris, Vuibert, coll. «Espace éthique».

APOLLINAIRE, Guillaume (2007). Si je mourais làbas, Paris, Complexe.

ASSAF, Francis (2006). "Philosophies et visions de la mort dans le premier âge baroque», dans Papers on French Seventeenth Century Literature, vol. 33, n 65, p. 403-418.

BATTIN, Margaret Pabst (2005). Ending Life. Ethics and the way we die, Oxford, Oxford University Press.

BAUDRY, Patrick (2006). La place du mort. Enjeux et rites, Paris, L'Harmattan, coll. « Nouvelles études anthropologiques».

BEAUCHEMIN, Jean-François (2005). La fabrication de l'aube, Montréal, Québec Amérique.

BERREGARD, Sandrine (2006). "Le spectacle de la mort et le problème des bienséances dans L'Hypochondriaque ou le mort amoureux et I'Hercule mourant de Rotrou", dans Papers on French Seventeenth Literature, vol. 33, $\mathrm{n}^{\circ} 65$, p. 193-206.

BERTRAND, Régis, CAROL, Anne et PELEN, JeanNoël (dir.) (2005). Les narrations de la mort, Aix-en-Provence, Publications de l'Université de Provence, coll. "Le temps de l'histoire».

BIANCHI, Enzo (2005). Vivre la mort, Paris, Parole et silence.

BIDENT, Christophe (2005). « Reconnaître la mort», dans Lettres Romanes, numéro spécial: « Maurice Blanchot, la singularité d'une écriture », p. 45-58.

BINET-SANGLE, Charles (2007). L'art de mourir. Défense et technique du suicide secondé, Paris, L'Harmattan, coll. «Thanatologie».

BOIA, Lucian (2006). Quand les centenaires seront jeunes. L'imaginaire de la longévité, de l'Antiquité à nos jours, Paris, Les Belles Lettres.

BOURGET, Jean-Loup (2005). "Question de vie ou de mort: la recherche d'une forme pour la mort", dans Positif: revue mensuelle de cinéma, $n^{\circ} 532$ (juin), p. 104-106.

BRODKEY, Harold (2001). Histoire de ma mort, Paris, Le Livre de Poche.

CASTA, Isabelle (2007). Nouvelles mythologies de la mort, Paris, Honoré Champion, coll. «Bibliothèque de littérature générale et comparée ».

CHEN, Ying (2006). Le Mangeur, Montréal, Boréal.

CHONG, Wang (2006). De la mort, Paris, Gallimard, coll. «Folio $2 €$ ».

COLLECTIF (2007). Devant la mort. Textes non bibliques pour les funérailles, Ivry-sur-Seine, Éditions de l'Atelier, coll. "Vivre Croire Célébrer ».

CONCHE, Marcel (2007). La mort et la pensée, Nantes, Cécile Defaut.
CROFT, Esther (2007). Le reste du temps, Montréal, $X Y Z$, coll. «Romanichels».

CUCHET, Guillaume (2005). Le crépuscule du purgatoire, Paris, Armand Colin, coll. "Histoire à l'œuvre».

DALAÏ-LAMA (2007). Vaincre la mort, vivre une vie meilleure, Paris, J'ai lu.

DARRAS, Jacques (2006). Nous ne sommes pas faits pour la mort, Paris, Stock, coll. "L'autre pensée".

DASTUR, Françoise (2005). Comment affronter la mort?, Paris, Bayard, coll. "Temps d'une question $"$.

DAYAN ROSENMAN, Anny (2007). Les alphabets de la shoah: survivre, témoigner, écrire, Paris, CNRS, coll. «CNRS histoire».

DEBOUT, Michel et CETTOUR, Denis (2006). Science et mythologie du mort, Paris, Vuibert, coll. «Culture scientifique».

DOLTO, Catherine (2006). Si on parlait de la mort, Paris, Gallimard, coll. «Mine de rien ».

DUTOURD, Jean (2006). Journal intime d'un mort, Paris, Pocket.

EICHEL-LOJKINE, Patricia et MARTIN-ULRICH, Claude (dir.) (2006). De bonne vie s'ensuit bonne mort: récits de mort, récits de vie en Europe (XVe$X V I I$ siècles), Paris, Honoré Champion, coll. "Colloques, congrès et conférences sur la littérature européenne».

EPHRAÏM (2006). Réenchanter la mort. Ma vie avec les morts, Longué-Jumelles (Maine-et-Loire), Arsis, coll. «Réenchanter sa vie».

ESON, Margaret (2000). Un trait de l'esprit, Arles, Actes Sud, coll. «Papiers».

ESTEBAN, Claude (2007). La mort à distance, Paris, Gallimard, coll. «Blanche ».

ÉTALON, Michel (2005). La survie ou le néant, Inguiniel, Malourène.

FORT, Pierre-Louis (2007). Ma mère, la morte. L'écriture du deuil chez Yourcenar, Beauvoir et Ernaux, Paris, Imago.

FOURNIER, Michel (2006). " "Allons allègrement mourir en philosophe" : De la mort du philosophe à la mort du libertin ", dans Papers on French Seventeenth Century Literature, vol. 33, $n^{\circ} 65$, p. 389-401.

FRANCGUR, Marie et Louis (2006). Plus fort que la mort. Récit-témoignage, Paris, Cerf.

FROMAGET, Michel (2007). Naître et mourir. Anthropologie spirituelle des mourants, Paris, Éd. François-Xavier de Guibert, 2007.

GILBERT, Christian (2007). Si je meurs, je veux qu'on m'empaille, Paris, Éditions Complicités.

HADJADJ, Fabrice (2005). Réussir sa mort: antiméthode pour vivre, Paris, Presses de la Renaissance.

HÉBERT, François (2007). Comment serrer la main de ce mort-là, Montréal, Hexagone, coll. «L'appel des mots ".

HENNIG, Jean-Luc (2007). Morgue. Enquête sur le cadavre et ses usages, Paris, Gallimard, coll. «Verticales».
HENNEZEL, Marie (de) (2007). Mourir les yeux ouverts, Paris, Pocket.

ID. et MITTERAND, François (2006). La mort intime. Ceux qui vont mourir nous apprennent à vivre, Paris, Pocket.

HIGGINS, Robert William, RICOT, Jacques et BAUDRY, Patrick (2006), Le mourant, Vallet, Éditions M-Éditer.

HIRSCH, Emmanuel (dir.) (2006). Face aux fins de vie et à la mort. Éthique, société, pratiques professionnelles (nouvelle édition augmentée), Paris, Vuibert, coll. « Espace éthique».

JACQUET-SMAILOVIC, Murielle (2006). Avant que la mort ne nous sépare: patients, familles et soignants face à la maladie, Bruxelles, De Boeck, coll. «Comprendre».

JANKÉLÉVITCH, Vladimir (1990). La Mort, Paris, Flammarion, coll. «Champs».

ID. (2003). Penser la mort?, Paris, Liana Levi, coll. "Piccolo».

JUNG, Young-Moon (2007). Pour ne pas rater ma dernière seconde. Récits d'outre-noir, Montréal, $X Y Z$, coll. «Romanichels».

KAREH TAGER, Djénane (2007). La vie après la mort, Paris, Plon, coll. "Petite bibliothèque Spirituali ».

KIAROSTAMI, Abbas (2002). Le vent nous emportera, Paris, Cahiers du Cinéma Livres, coll. «Petite bibliothèque des Cahiers du cinéma ».

KIERKEGAARD, Søren (2007). La maladie à la mort (Traité du désespoir), Paris, Nathan, coll. "Les intégrales de philo».

KRISTEVA, Julia, MICHEL, Régis et VIATTE, Françoise (1998). Visions capitales, Paris, Réunion des musées nationaux.

KRIVOPISSKO, Guy (2006). La vie à en mourir, Paris, Seuil, coll. «Points Histoire ».

LARGO, Michael (2006). Final Exits: The Illustrated Encyclopedia of How we Die, New York, Harper.

LAVOCAT, François et LECERCLE, François (dir.) (2005). Dramaturgies de I'ombre. Actes du colloque organisé à Paris IV et Paris VII, 27 au 30 mars 2002, Rennes, Presses universitaires de Rennes, coll. «Interférences».

LEADBEATER, Charles Webster (2007). L'autre côté de la mort, Paris, Adyar.

LE BRAZ, Anatole (2006). La légende de la mort chez les Bretons armoricains, Rennes, Terre de brume, coll. «Bibliothèque celte».

LE COZ, Pierre (2006). Le médecin et la mort. Approches éthiques et philosophiques, Paris, Vuibert, coll. "Espace éthique».

LE GUAY, Damien (2003). Qu'avons-nous perdu en perdant la mort?, Paris, Cerf, coll. "Histoire à vif ».

LEIVA, Antonio Dominguez (2005). Décapitations. Du culte des crânes au cinéma gore, Paris, Presses universitaires de France, coll. «Littératures européennes». 
LENOIR, Frédéric, TONNAC, Jean-Philippe (de) et alii (2004). La mort et l'immortalité. Encyclopédie des savoirs et des croyances, Paris, Bayard.

LÉPINE, Jacques-Jude (1997). «De la pensée de la mort à la descente aux Enfers: Pour une étude d'ensemble sur Pascal et le christianisme orthodoxe", dans Papers on French Seventeenth Century Literature, vol. 24, n 46, p. 175-187.

LE ROUX-KIEKEN, Aude (2005). Imaginaire et écriture de la mort dans l'œuvre de Marcel Proust, Paris, Honoré Champion, coll. «Recherches proustiennes".

LEVINAS, Emmanuel (2006). Altérité et transcendance, Paris, Librairie Générale Française / Hachette, coll. "Le Livre de poche Biblio essais ».

ID. (2006). La mort et le temps, Paris, L'Herne, coll. "Les cahiers de l'Herne ».

MAREAU, Charlotte (2007). Parler de la mort, Levallois-Perret, Studyrama, coll. «Éclairages».

MICHAUD NÉRARD, Michel (2007). La révolution de la mort. Pompes funèbres aux services funéraires, Paris, Vuibert, coll. "Espace éthique».

MOLINIÉ, Magali (2006). Soigner les morts pour guérir les vivants, Paris, Le Seuil et Les Empêcheurs de penser en rond.

MOODY, Raymond, LOMMEL, Pim (van), PARNIA, Sam et alii (2007). L'expérience de mort imminente. Actes du colloque tenu à Martigues le 17 juin 2006, [sans lieu], Éditions S17.

MPONIMPA, Melchior (2006). Les morts ne sont pas morts, Sudbury, Prise de Parole.

MUMFORD, John (2006). Mourir, une fin ou un début. Méthode vers l'immortalité, Noisy-surÉcole, Éditions de l'Éveil.

Nouvel Observateur (avril-mai 2006, numéro hors série) «Apprivoiser la mort pour mieux vivre».

NUTKOWICZ, Hélène (2006). L'homme face à la mort au royaume de Juda, Paris, Cerf, coll. "Patrimoines Judaïsme ».

OSHO (2006). Un art de vivre et de mourir, Gordes, Éditions du Relié, coll. «Relié poche».

PAVIOT, Christophe (2007). Devenir mort, Paris, Hachette, coll. «La Fouine».

PERROT-CORPET, Danielle (2005). Écrire devant I'absolu. Georges Bernanos et Miguel de Unamuno, Paris, Honoré Champion, coll. «Bibliothèque de littérature générale et comparée ».

PIETTE, Albert (2005). Le temps du deuil: essai $d^{\prime}$ anthropologie existentielle, Ivry-sur-Seine, Éditions de l'Atelier.

PILLET, Claude (2006). "Le miroir des limbes: l'autre de la mort", dans Revue André Malraux Review, vol. 33, n 1, p. 52-59.

PHILLIPS, Adam (2005). De la mort qui fait aimer la vie: Darwin et Freud, Paris, Payot et Rivages, coll. «Petite bibliothèque Payot».

PRIGENT, Christian (2007). Demain je meurs, Paris, P.O.L.

QUILLIOT, Roland (2000). Qu'est-ce que la mort?, Paris, Armand Colin, coll. «U».
RICFUR, Paul (2007). Vivant jusqu'à la mort, suivi de Fragments, Paris, Seuil, coll. "Couleur des idées".

SAFTY, Essam (2005). La mort tragique. Idéologie et mort dans la tragédie baroque en France, Paris, L'Harmattan.

SALEM, Gilbert (2006). À la place du mort, Orbe, Éd. Bernard Campiche.

SAUL, Nicholas (2006). "Morbid? Suicide, Freedom, Human Dignity and the German Romantic Yearning for Death", dans Historical Reflections IRéflexions Historiques, vol. 33, n 3 (automne), p. 579-599.

SCARRE, Geoffrey (2007). Death, Montréal, McGillQueen's University Press, coll. "Central Problems of Philosophy ».

SCHUMACHER, Bernard N. (2005). Confrontations avec la mort. La philosophie contemporaine et la question de la mort, Paris, Cerf, coll. «Passages».

SCHWAB, Françoise (1995). "Penser la mort», dans: Magazine littéraire, $n^{\circ} 333$ (juin), p. 43-45.

SMITH, Wesley J. (2006). Forced Exit: Euthanasia, Assisted Suicide, and the New Duty to Die, New York, Encounter Books.

SINGER, Christiane (2007), Derniers fragments d'un long voyage, Paris, Albin Michel.

TEULÉ, Jean (2007). Le magasin des suicides, Paris, Julliard.

THEVOZ, Michel (2004). L'esthétique du suicide, Paris, Minuit, coll. "Paradoxe».

TILSON, Edward (2006). «Vers une eucharistie de melons: l'être, la mort et l'écriture dans l'essai III, 13 de Montaigne ", dans: Versants: Revue Suisse des Littératures Romanes, vol. 51, p. 5-63.

TOLSTOÏ, Léon (2006). La mort d'Ivan Ilitch, Paris, Librio.

URBAIN, Jean-Didier (2005). L'archipel des morts: cimetières et mémoire en Occident (édition revue et augmentée), Paris, Payot, coll. «Petite bibliothèque Payot ".

VATZBED, Éric (2005). Lumière d'outre-tombe, Paris, Presses universitaires de France, coll. «Perspectives critiques ».

WATTEYNE, Nathalie (2006). «Voix d'outretombe dans les poèmes en prose $d$ 'Isabelle Pinçon et de Louise Dupré ", dans BROPHY, Michael et GALLAGHER, Mary (dir.), Sens et présence du sujet poétique: la poésie de la France et du monde francophone depuis 1980, Amsterdam, Rodopi, p. 339-346.

WILLOCK, Brent, BOHM, Lori C. et Rebecca C. CURTIS (dir.) (2007). On Deaths and Endings: Psychoanalysts' Reflections on Finality, Transformations, and New Beginnings, Londres et New York, Routledge.

YONNET, Paul (2006). Le recul de la mort. L'avènement de l'individu contemporain, Paris, Gallimard, coll. «Bibliothèque des sciences humaines».

ZECH, Emmanuelle (2006). Psychologie du deuil: impact et processus d'adaptation au décès, Sprimont, Mardaga, coll. "Pratiques psychologiques». 